\title{
Trendelenburg Gait Improved by Trigger Point and Scar Tissue Lidocaine Injections: A Case Report
}

\author{
Alexander King, DO ${ }^{1,2^{*}}$, Rosie Wenrich, $B S^{2}$, Rachel Patel, $B S^{2}$ and Joseph LaFontaine, $B S^{2}$ \\ ${ }^{1}$ Rowan Medicine, NeuroMusculoskeletal Institute, Stratford, NJ, USA \\ ${ }^{2}$ Rowan University School of Osteopathic Medicine, Stratford, NJ, USA
}

*Corresponding author: Alexander King, D.O, NeuroMusculoskeletal Institute, Rowan Medicine, 1474 Tanyard Road, Suite A, Sewell, NJ 08080, USA, Tel: 856-566-7010, Fax: 856-566-6961

\begin{abstract}
This case report describes a 74-year-old Caucasian female patient who presented with bilateral gluteus muscle trigger point pain, decreased gluteus medius strength, a substantial transverse abdominal scar, and a history of knee replacement culminating in a Trendelenburg gait and chronic pain. Treatment of the patients' symptoms with lidocaine solution injections, known as neural therapy, into the bilateral gluteus muscle trigger points and along scar tissue facial planes yielded markedly improved gait and immediate resolution of trigger point pain. Neural therapy used in this patient, involves the injection of anesthetic solution into trigger points and scar tissue, to release muscle tension and encourage resetting of dysfunctional nerve pathways
\end{abstract}

\section{Keywords}

Trigger point, Neural therapy, Lidocaine injection, Trendelenburg gait, Scar tissue

\section{Key Message}

This case illustrates the efficacy of anesthetic injections into subcutaneous tissue and muscle to resolve chronic pain and musculoskeletal dysfunction. It elucidates neural therapy as a viable alternative to invasive surgical treatments for chronic pain with concurrent hypertrophic scar tissue resulting in nerve entrapment.

\section{Introduction}

Myofascial pain syndrome (MPS) is characterized by tender areas referred to as "trigger points" in muscle and connective tissue. These trigger points are often associated with muscle spasm, muscle stiffness, limited range of motion, loss of strength, and autonomic dysfunction. MPS is the most common cause of chronic musculoskeletal pain and can affect all aspects of a patient's life: often leading to opioid dependence, reduced ability to complete activities of daily living, anxiety and depression, and poor quality of life [1-3]. As technology advances, innovative therapies and surgical techniques are utilized to control chronic pain, however this can often be costly and invasive. As a result, more medical professionals and patients are beginning to investigate alternative forms of medicine that have been proven to effectively control chronic pain.

Neural therapy is a minimally invasive procedure that has been used for over a century to address myofascial pain syndrome and other forms of chronic pain $[4,5]$. Developed in the 1900's in Germany, this technique has recently gained popularity amongst medical communities throughout Europe and the United States [4]. Neural therapy involves injecting local anesthetics into scar tissue, trigger points, tendons, ligaments, and tissue in areas affected by chronic pain to release muscle tension and reset dysfunctional nerve firing. This disruption allows for the local muscle fibers to relax and for the nervous pathways to reestablish their proper trajectory $[1,2,6]$. Neural therapy can elicit immediate results including increased range of motion and decreased trigger point pain. It has also been proven to have lasting effects $[1,3,6]$.

Alternate methods to neural therapy include dry needling and botulism injections. Dry needling involves penetrating the dysfunctional tissue with empty syringes

Citation: King A, Wenrich R, Patel R, LaFontaine J (2022) Trendelenburg Gait Improved by Trigger Point and Scar Tissue Lidocaine Injections: A Case Report. Clin Med Rev Case Rep 9:382. doi. org/10.23937/2378-3656/1410382

Accepted: January 29, 2022: Published: January 31, 2022

Copyright: (c) 2022 King A, et al. This is an open-access article distributed under the terms of the Creative Commons Attribution License, which permits unrestricted use, distribution, and reproduction in any medium, provided the original author and source are credited. 
to mechanically disrupt the region, while botulism injections include injecting 10-20 IU of BTX solution into the tissue [2]. A study comparing the three methods found that lidocaine injections and BTX injections yielded a significant decrease in pain, fatigue, and work disability at one month post-injection treatment, while dry needling did not show any significant change in these markers. Dry needling also resulted in the most discomfort post-treatment, with $80 \%$ of patients reporting pain at the time of treatment, compared to $20 \%$ in the lidocaine treatment group and $0 \%$ in the BTX treatment group [2]. One month post-treatment, the lidocaine injection group maintained the lowest pain score. This result, along with the cost effectiveness of anesthetic treatment compared with BTX treatment, indicates lidocaine injections as a first-choice treatment for myofascial pain syndrome $[2,4]$.

\section{Case Presentation}

The patient is a 74-year-old female presenting to the Neuromuscular Institute to address chronic bilateral hip pain and a severe gait dysfunction. The patient has a long history of knee joint pain and dysfunction and received Synvisc injections to her knees bilaterally in 2018. She also underwent a right knee replacement in 2019. The patient reports that her gait deteriorated approximately one year following her Synvisc injection treatment in 2018 and then worsened further following her knee replacement. The patient reports that it is around this time that she began experiencing severe bilateral hip pain that is currently more severe on the left side. The patient has seen multiple healthcare providers and has completed 17 weeks of physical therapy to address these issues with no success. The patient reports that her hip pain is exacerbated by physical movement and is relieved by rest. She denies any numbness or tingling. The patient also relies heavily on a cane when ambulating.

\section{Past surgical history}

The patient is gravida 3 para 3 via cesarean section. She underwent panniculus removal with a graft placement in 1995, resulting in an enlargement of the lower abdominal scar. The patient also had a right knee replacement in 2019.

\section{Physical exam results}

The patient walks with a left leaning Trendelenburg gait and uses a cane for ambulation. There was $4 / 5$ muscle strength in bilateral gluteus medius, $5 / 5$ muscle strength in quadriceps, hamstrings, triceps, biceps bilaterally. There was an absent right patellar deep tendon reflex,+3 left patellar deep tendon reflex. There were also +2 bilateral biceps and brachioradialis deep tendon reflexes. The patient has a substantial transverse abdominal scar (Figure 1) from the right to left oblique, approximately 2 feet in length, with dimpling and hypertrophic regions, indicating fascial attachments and possible adhesion to underlying musculature and visceral organs. The patient also has a sagittal right knee scar approximately 7 inches in length. The patient also complains of tenderness and active trigger points in the gluteus minimus bilaterally with radiation into the hips.

\section{Differential diagnosis}

In order to target the patient's specific pain, it is

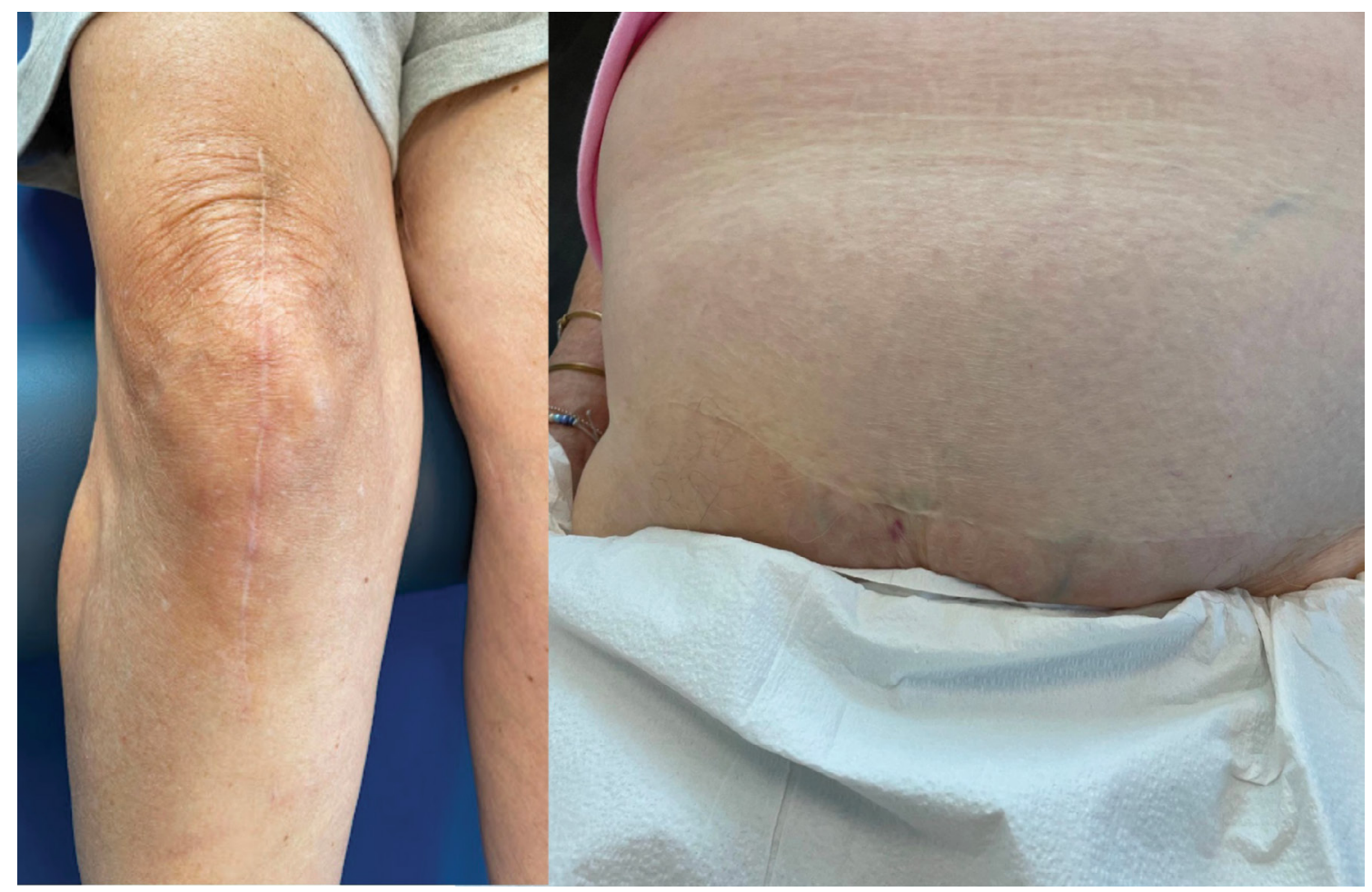

Figure 1: Left: Knee replacement scar, Right: Panniculectomy scar. 
important to note that the patient's gait dysfunction has multiple etiologies including gluteus medius and maximus weakness, active trigger points in hips, and scar tissue interference. Nerve entrapment in hypertrophic scar tissue and muscle weakness are causing the patient to walk with a Trendelenburg gait.

\section{Treatment}

It was my clinical decision to use lidocaine injections to disrupt the trigger points and promote relaxation and lengthening of muscle, improve range of motion, allow for scar tissue release, decrease adhesions, and improve nerve conduction through the affected region. On day one of her treatment, the patient laid in a prone position and the target tissue was palpated to locate exquisitely tender areas that elicit a muscle twitch and cause a referred pain pattern. The area was prepped, and these trigger points were marked. A 25-gauge needle was used to inject a $5 \mathrm{cc}$ mixture of $1 \%$ lidocaine and saline through the skin and subcutaneous tissue into a taut left gluteus medius and a left gluteus maximus. She was also recommended supportive footwear to improve her gait dysfunction as an adjunctive lifestyle modification.

On day 10, during her follow-up therapy, the patient lay supine, and a 25-gauge needle was used to inject a $5 \mathrm{cc}$ mixture of $1 \%$ Lidocaine and saline through skin and subcutaneous tissue along the bilateral taut oblique muscles and the right vastus medialis. The same injection mixture was then used to treat the large abdominal scar by injecting into the subcutaneous facial planes along the entire scar line. Finally, the same mixture was used to inject the subcutaneous tissue along the length of the patient's knee replacement scar.

\section{Outcome}

The patient reported complete resolution of trigger point related pain in the gluteus muscles immediately following injections. There was significant objective improvement in gait and range of motion immediately after the first set of injections, resulting in about 50\% less drop in patient's right pelvis and left leaning compensation. Ten days post-injection treatment, the patient returned to the office with new supportive footwear and only a mild objective slouching of the hip towards the right during the right leg swing phase. Post second set of injections patient showed immediate results with improved gait indicated by approximately $80 \%$ improvement in both the right pelvis drop and the left leaning as compared to initial observation on patient's first visit. Patient's hip flexor range of motion was greatly improved following abdominal scar injections. On her way out of the office, she was no longer relying on her cane to ambulate at all. One day post second treatment, the patient reported muscle pain in the hip contralateral to treatment. One month post injections, patient reported to follow up appointment and reported that she is ambulating independently and no longer relying on a cane at all.

\section{Discussion}

We hypothesize that the abundance of hypertrophic scar tissue in the patient's lower abdomen from her prior panniculus removal was causing compression of the superior gluteal nerve against the pelvic wall, resulting in the observed Trendelenburg gait [7]. We also theorize that the scar tissue was causing interference with the nervous pathway from the pelvic region to the lower extremities [7,8]. In addition, we speculate that range of motion of the hip flexors and quadricep muscles were limited due to repeated knee trauma. The lidocaine injections yielded immediate results because it allowed for relaxation of the taut muscles and trigger points of the affected lower extremity. Further improvement was observed 10 days post injection as the dysfunctional nerve communication that had been previously evident within the hypertrophic scar tissue was reset by sodium channel blockade, thus gaining increased communication to their target muscles in the lower extremity. It is expected for these improvements to remain, with the possible need for occasional maintenance injections.

We postulate that the new onset of pain following the second treatment is a result of the body adapting to musculoskeletal changes on the left. As the patient's dysfunctional muscles regain mobility and strength, the musculature that had been compensating for many years must adapt to the sudden change. It is also expected that the patient will sometimes experience soreness that can last 24 to 48 hours post-treatment. The patient was directed to use a heating pad or a hot compress as needed, as well as over-the-counter pain medications on the affected area to provide some relief.

As illustrated by this case, neural therapy is a minimally invasive, cost-effective procedure that can result in substantial improvements in musculoskeletal dysfunctions. In similar cases of nerve entrapment, treatment options were previously believed to be limited to manual stretching therapies or invasive surgical methods such as neurolysis or neurectomy $[9,10]$. This case elucidates the importance of neural therapy as a treatment option for myofascial pain syndrome and scar tissue nerve entrapment (Table 1 ). In the patient's words, "this treatment has been the first one to give me any sort of real relief. I am almost walking normally. I think it's been really effective so far and I'm going to keep up with it. I don't think I'm 100\% there yet but I think [my pain and gait] will keep improving. I'm not using my cane anymore and I can definitely feel myself walking much better."

\section{Note on Patient Consent}

Informed written consent was obtained from the patient to conduct this case report and to include photographs. 
Table 1: Historical and current timeline of care.

\begin{tabular}{|c|c|c|}
\hline Dates & Event & Outcome \\
\hline $\begin{array}{l}1967,1982 \\
1984\end{array}$ & 3 Cesarean Section Deliveries & Abdominal Scarring \\
\hline 1995 & Panniculectomy & $\begin{array}{l}\text { Expansion of abdominal scar, Trendelenburg gait } \\
\text { began to develop }\end{array}$ \\
\hline 2018 & Synvisc Knee Injections & Trendelenburg gait exacerbation \\
\hline 2019 & Right Knee Replacement & Trendelenburg gait exacerbation \\
\hline 2020 & 17 Weeks of Physical Therapy & No improvement \\
\hline $\begin{array}{l}\text { August 3, } \\
2021\end{array}$ & $\begin{array}{l}1^{\text {st }} \text { Lidocaine Injections into gluteus medius, gluteus } \\
\text { maximus }\end{array}$ & $\begin{array}{l}\text { Immediate improvement in gait and resolution of } \\
\text { trigger point pain }\end{array}$ \\
\hline $\begin{array}{l}\text { August 13, } \\
2021\end{array}$ & $\begin{array}{l}2^{\text {nd }} \text { Lidocaine Injections into oblique muscles, vastus } \\
\text { medialis, panniculectomy scar, knee replacement scar }\end{array}$ & $\begin{array}{l}\text { Resolution of trigger point pain, improved range of } \\
\text { motion, improved gait }\end{array}$ \\
\hline
\end{tabular}

\section{Conflicts of Interest}

None.

\section{Funding Source}

None.

\section{References}

1. Brobyn TL, Chung MK, LaRiccia PJ (2015) Neural therapy: An overlooked game changer for patients suffering chronic pain. J Pain Relief 4: 184.

2. Kamanli A, Kaya A, Ardicoglu O, Ozgocmen S, Zengin FO, et al. (2005) Comparison of lidocaine injection, botulinum toxin injection, and dry needling to trigger points in myofascial pain syndrome. Rheumatol Int 25: 604-611.

3. Wong CSM, Wong SHS (2012) A new look at trigger point injections. Anesthesiol Res Pract 2012: 492452.

4. Hong CZ (1994) Lidocaine injection versus dry needling to myofascial trigger point. The importance of the local twitch response. Am J Phys Med Rehabil 73: 256-263.
5. Lemos N, Sermer C, Fernandes G, Morgado-Ribeiro A, Rossos A, et al. (2021) Laparoscopic approach to refractory extraspinal sciatica and pudendal pain caused by intrapelvic nerve entrapment. Sci Rep 11: 10820.

6. Harris GR (2010) Effective treatment of chronic pain by the integration of neural therapy and prolotherapy. Journal of prolotherapy 2: 377-386.

7. Ulloa M, Banda MC (2017) Scar tissue causing saphenous nerve entrapment: Percutaneous scar release and fat grafting. Plast Reconstr Surg Glob Open 5: e1495.

8. Murat S, Altınbilek T (2019) Is chronic pain related with the postsurgical scar tissue in women? Med Med J 34: 47-53.

9. Martin R, Martin HD, Kivlan BR (2017) Nerve entrapment in the hip region: Current concepts review. Int J Sports Phys Ther 12: 1163-1173.

10. Martin HD, Shears SA, Johnson JC, Smathers AM, Palmer IJ (2011) The endoscopic treatment of sciatic nerve entrapment/deep gluteal syndrome. Arthroscopy 27: 172181. 